\title{
Ultrastructural Morphology and Protein Content of the Internal Organic Material of Rat Otoconia
}

\author{
KenNeth G. Pote*,1 and Muriel D. Ross $\dagger$ \\ *The Department of Anatomy and Cell Biology, The University of Michigan, Ann Arbor, Michigan 48109, and \\ $\uparrow$ Space Biology Branch, NASA, Ames Research Center, Moffett Field, California 94035
}

Received July 18, 1986

\begin{abstract}
Otoconia taken from the maculae of the saccule and utricle of the rat inner ear were investigated using transmission electron microscopy (TEM). Their ultrastructure was compared using various decalcification techniques. Similar preparations were analyzed using sodium dodecyl sulfate-polyacrylamide gel electrophoresis (SDS-PAGE) to assess their protein constituents. TEM results show loss of organic material during prolonged decalcification. This is not seen in samples decalcified overnight. The SDS-PAGE results show that rat otoconia contain a major broad band of 90-100 $\mathrm{kDa}$, a major thin band of $56 \mathrm{kDa}$, and three other bands of 33,45, and $50 \mathrm{kDa}$. The hand of 56 $\mathrm{kDa}$ may be associated with the surface of the otoconia. (c) 1986 Academic Press, Inc.
\end{abstract}

The vestibular portion of the vertebrate inner ear contains the maculae of the saccule and utriclc. In mammals, macular end organs function as bioaccelerometers to detect linear acceleration. They consist of a sensory neuroepithelium overlain by an otoconial membrane within or upon which are many small otoconia. Otoconia are composites of organic and inorganic (mineral) materials, as has been recognized since the last century (Henle, 1873). In mammals, the mineral is calcium carbonate in the form of calcite. The precise composition of the organic phase is unknown, but it consists of protein and carbohydrate according to results from histochemical (Wislocki and Ladman, 1955; Belanger, 1960) and biochemical studies (Ross and Pote, 1984; Ross et al., 1985; Gil-Loyzaga et al., 1985).

Research using sodium dodecyl sulfatepolyacrylamide gel electrophoresis (SDSPAGE) showed that rat otoconial complexes (otoconia plus the otoconial membrane) contain many proteins in a range between 15000 and over $100000 \mathrm{Da}$ (Ross et al., 1981). Latcr results indicatcd a preponderance of acidic versus basic amino acids in otoconial proteins, the otoconial mem-

\footnotetext{
${ }^{1}$ To whom correspondence should be addressed.
}

brane having been removed by centrifugation (Ross and Pote, 1984). The results of the amino acid analyscs are comparable with those of Degens (1976), who analyzed the organic material of otoliths and neogastropod shells.

It is important to learn more about the organization and composition of the organic material because it is thought to be involved in the seeding, growth, and inhibition of the mineral crystallites. A central core and peripheral zone have been observed in mammalian otoconia by several investigators using light microscopy (Henle, 1873; Carlstrom et al., 1953; Lindeman, 1969) and electron microscopy (Marco et al., 1971; Lim, 1973; Salamat et al., 1980). Salamat et al., (1980) suggested that a relationship exists between the sizc of the corc and the ultimate size of an otoconium. A problem concerning the possible significance of the core is the variation in density of organic material observed by various investigators. Some (Salamat et al., 1981) have observed a great deal of organic material while others have seen very little (Igarashi and Kanda, 1969; Marco et al., 1971; Sanchez-Fernandez et al., 1972; Nakahara and Bevelander, 1979; and others). It is not known whether this is due to the method of 
fixation. Regardless, the presence of a central core and a peripheral zone indicates that at the very least there should be two organic materials in otoconia. Additionally, slightly different organic material may coat the otoconia, to inhibit or to limit their growth.

It is clear that neither the composition nor the distribution of the organic material of otoconia has been demonstrated definitively. The present study began as an attempt to clarify the ultrastructural appearance of the organic material after various decalcification methods and to correlate findings with protein distribution. Proteins were separated using sodium dodecyl sulfate-polyacrylamide gel electrophoresis. This resulted in identification of proteins present inside and outside the otoconia, and at the otoconial surface.

\section{MATERIALS AND METHODS}

Water was purified by reverse osmosis and ion-exchange polishing (Millipore Milli-Q). All chemicals were reagent grade. Animals were male $C D$ strain rats weighing between 100 and $125 \mathrm{~g}$ (Charles River). Centrifugation was done in a clinical centrifuge (IEC, Model CL) run at top speed.

\section{Decalcification, Staining, and Electron Microscopy}

Otoconia decalcified in situ. Rats were decapitated, the bullae were removed and, after opening the round and oval windows, the labyrinth was perfused with fixative using a syringe. The following methods were used.

(1) Eight animals were treated with $2 \%$ glutaraldehyde in modified Sorenson's buffer, pH 7.4. Half of these samples were decalcified with $5 \%$ formic acid (FA) overnight (modification of Ippolito et al., 1981) and half with $100 \mathrm{~m} M$ EDTA containing $4 \%$ glutaraldehyde for 30 days (solution changed every other day). Two of the formic acid decalcified inner ears were treated overnight with the EDTA solution following decalcification.

(2) In a second group of three rats, $4 \%$ tannic acid was added to the primary fixative in method (1), without altering the resultant $\mathrm{pH}$ of 6.4. This was an attempt to localize carbohydrate. These inner ears were decalcified overnight in 5\% formic acid containing $2 \%$ glutaraldehyde.

Following decalcification, tissues were washed three times in buffer followed by $1 \mathrm{hr}$ postfixation in $1 \%$ $\mathrm{OsO}_{4}$ in buffer at room temperature. The specimens were washed three times in buffer and dehydrated in a graded series of alcohol. All inner ears were embedded in Spurr's (1969) medium, polymerized, and sectioned using an AO-Reichert Ultracut E ultramicrotome. Thick sections were cut using glass knives until the saccule was reached. Blocks were precision trimmed and thin sectioned with a Diatome diamond knife. Sections were stained with uranyl acetate and with lead citrate. They were examined and photographed with a Zeiss EM 10A electron microscope.

Otoconia decalcified after microdissection. These samples were prepared for EM to ascertain what structural elements were present in the dissected samples prepared for SDS-PAGE. Following decapitation, the inner ear bullae were removed, and the otoconia from two animals per sample were microdissected in artificial endolymph, pH 7.4 (Ross et al., 1981) on ice and treated in one of the following ways:

(1) Placed in a flat embedding mold for decalcification with either (a) 5\% EDTA + 4\% glutaraldehyde for $1 \mathrm{hr}$ or (b) $4 \%$ glutaraldehyde for $1 \mathrm{hr}$, quenched with $1 \% \mathrm{NaBH}_{4}$ for $25 \mathrm{~min}$, and decalcified with $5 \%$ EDTA overnight.

(2) Placed in 1.5-ml conical microcentrifuge tubes, sonicated for $5 \mathrm{~min}$ in $25 \mu \mathrm{l} 2 \%$ SDS in $0.1 \mathrm{M}$ sodium acetate ( $\mathrm{pH} 7.6$ ), and centrifuged for $5 \mathrm{~min}$. This was repeated three times. Samples were then decalcified for $1 \mathrm{hr}$ with $100 \mathrm{~m} M$ EDTA in the presence of $4 \%$ glutaraldehyde.

These samples were then embedded, sectioned, stained, and examined in the electron microscope as described above.

FIG. 1. Dissected otoconium decalcified with $5 \%$ EDTA $+4 \%$ glutaraldehyde. The otoconial membrane is present and the internal otoconial matrix is intact and segmented. om, otoconial membrane. Bar $=1.0 \mu \mathrm{m}$.

FIG. 2. Otoconia decalcified 30 days in situ with $100 \mathrm{~m} M$ EDTA $+4 \%$ glutaraldehyde. The external lamina and the segmented appearance are absent, while the inner material and the central core are preserved. Bar $=$ $1.0 \mu \mathrm{m}$.

Fig. 3. Otoconia decalcified in situ with $100 \mathrm{~m} M$ EDTA $+4 \%$ glutaraldehyde. The edges are indistinct and the internal material is somewhat granular in appearance. Bar $=1.0 \mu \mathrm{m}$.

FIG. 4. Otoconia treated in situ with EDTA after decalcification with $5 \%$ formic acid $+4 \%$ glutaraldehyde. This dense core is unusually large. Bar $=1 \mu \mathrm{m}$. 
PROTEINS OF RAT OTOCONIAL COMPLEX

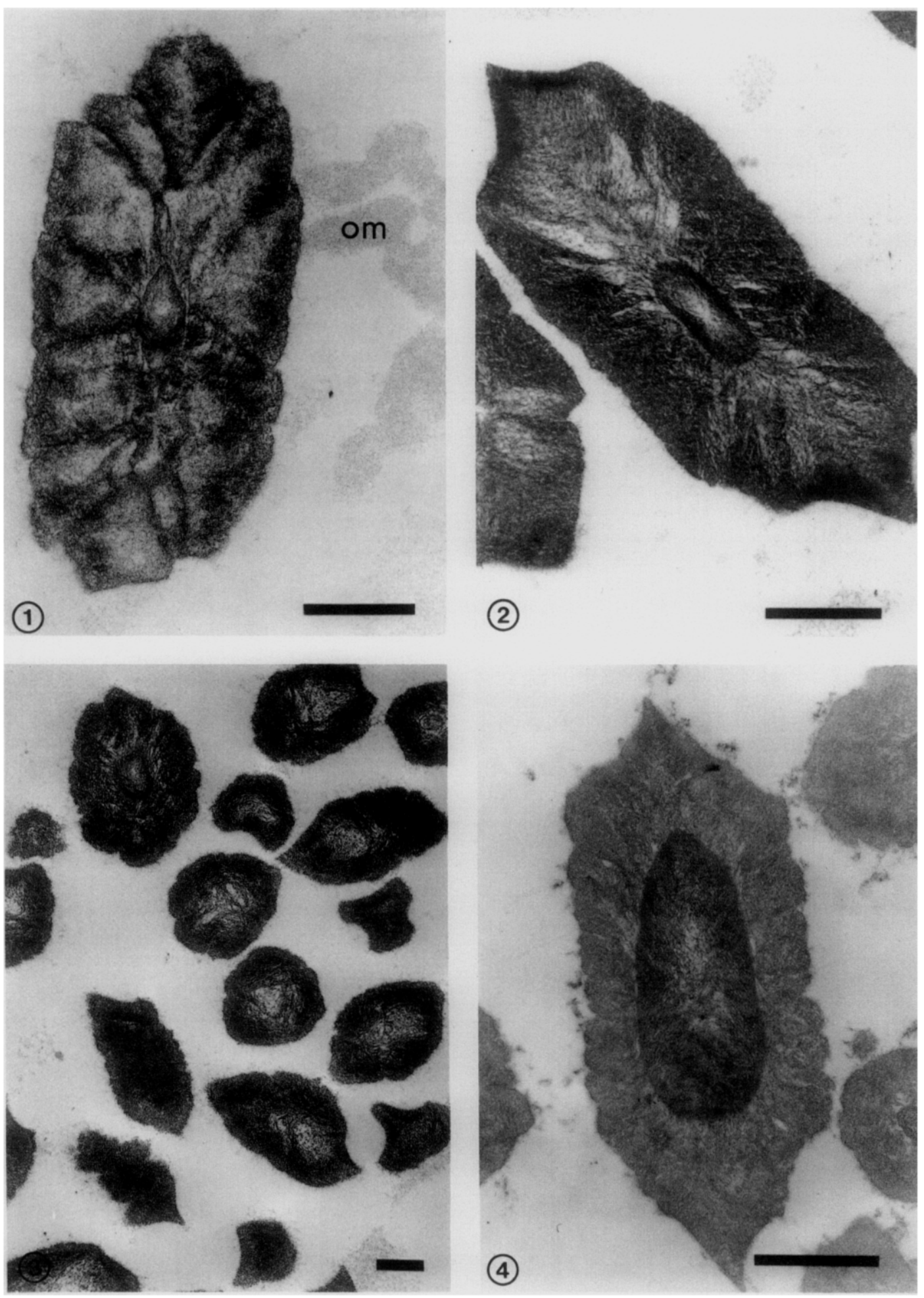


SDS Gel Electrophoresis

Polyacrylamide gels of $15 \times 15 \times 0.75 \mathrm{~cm}$ were cast using a modification of Anderson and Anderson (1978) methods for casting multiple gels. The running gels were linear gradients from 6.0 to $16.6 \%$ with a $4 \%$ stacking gel. Laemmli's (1970) Tris-glycine buffer system was used throughout. Otoconia from the saccule and utricle of four animals were microdissected in artificial endolymph on ice and combined in a $1.5-\mathrm{ml}$ microcentrifuge tube for each sample. The samples were centrifuged to pellet the otoconia. These samples were treated in one of the following ways:

(1) Decalcified in $25 \mu \mathrm{l} 5 \%$ EDTA for $1 \mathrm{hr}$.

(2) Sonicated in $25 \mu \mathrm{l} 0.1 \mathrm{M}$ sodium acetate (pH 7.6) plus or minus $2 \%$ SDS, and then centrifuged. This was repeated three times, and then the sample was treated as in sample (1). This was done to separate the proteins within the otoconia from those of the otoconial membrane (Ballarino, 1985) and to ascertain whether SDS is required for this separation.

(3) Treated with $25 \mu 12 \%$ glutaraldehyde for $1 \mathrm{hr}$, centrifuged and supernatant removed, quenched with $25 \mu 11 \% \mathrm{NaBH}_{4}$, centrifuged and supernatant combined with the previous, and then both were treated as sample (1) above. This treatment should cross-link the proteins outside the otoconia and leave those inside relatively unaffected.

(4) Decalcified in $25 \mu \mathrm{l} 5 \%$ EDTA in the presence of $2 \%$ glutaraldehyde followed by centrifugation as in sample (3) above. This will mimic the treatment of the ultrastructural study. This treatment should cross-link the proteins inside the otoconia as they are exposed during decalcification.

All these samples were then treated with $25 \mu \mathrm{l} 2 \mathrm{X}$ sample buffer; IX consists of $10 \% \mathrm{v} / \mathrm{v}$ glycerol, $5 \% \mathrm{v} / \mathrm{v}$ 2-mercaptoethanol, $2.3 \% \mathrm{w} / \mathrm{v}$ sodium dodecyl sulfate, and $0.625 \mathrm{M}$ Tris- $\mathrm{HCl}$. They were then heated in a boiling water bath for $10 \mathrm{~min}$. Twenty-five microliters of samples thus prepared are adequate for detection with the silver staining method of Heukeshoven and Dernick (1985).

\section{RESULTS}

\section{Electron Microscopy}

EDTA decalcification. Different decalcification techniques produce different otoconial ultrastructure. Overnight decalcification of dissected otoconia in EDTA retains a well-defined central core, peripheral zone, and external lamina (Fig. 1). The organic material of the central core and the peripheral zone is not distributed uniformly. It is more dense at the border of the central core and near the surface of the otoconium. Fibrillar-appearing organic material extends from the core's border to the surface. This gives the peripheral zone a segmented appearance. By contrast, prolonged (30 days) decalcification in EDTA does not always retain the external lamina (Figs. 2 and 3). Additionally, the organic material is more condensed around the central core and in the peripheral zone so that a segmented appearance is obscure.

Formic acid decalcification. Overnight decalcification in FA followed by short exposure to EDTA results in preservation of all organic components of otoconia. In some cases the central cores are very apparent and of great size (Fig. 4). By contrast, FA decalcification alone causes the loss of some organic material (Fig. 5), and the central core is not evident in the majority of otoconia. It should be noted that otoconia must be sectioned through their centers to visualize the core (Fig. 2).

Tannic acid. Inclusion of tannic acid in

Fig. 5. Otoconia decalcified in situ with $5 \%$ formic acid $+4 \%$ glutaraldehyde. The internal material is well prescrved as is the surface lamina. The majority of the otoconia lack a central core. Bar $=1.0 \mu \mathrm{m}$.

FIG. 6. Otoconia treated in situ with tannic acid and decalcified with $5 \%$ formic acid $+4 \%$ glutaraldehyde. The otoconia contact the otoconial membrane and retain their internal material. A granular substance is above the otoconia. Bar $=1.0 \mu \mathrm{m}$.

FIG. 7. Otoconia treated in situ with tannic acid and decalcified with $5 \%$ formic acid $+4 \%$ glutaraldehyde. The dark-stained external lamina is evident, as is a fine line of material just internal to it. Bar $=0.2 \mu \mathrm{m}$.

FIG. 8. Dissected otoconia sonicated and centrifuged in the presence of $2 \%$ SDS, decalcified with $5 \%$ EDTA + $4 \%$ glutaraldehyde. This sample had very little organic material between the otoconia and no cellular debris, but lost the external lamina and internal segmentation. Bar $=1.0 \mu \mathrm{m}$. 

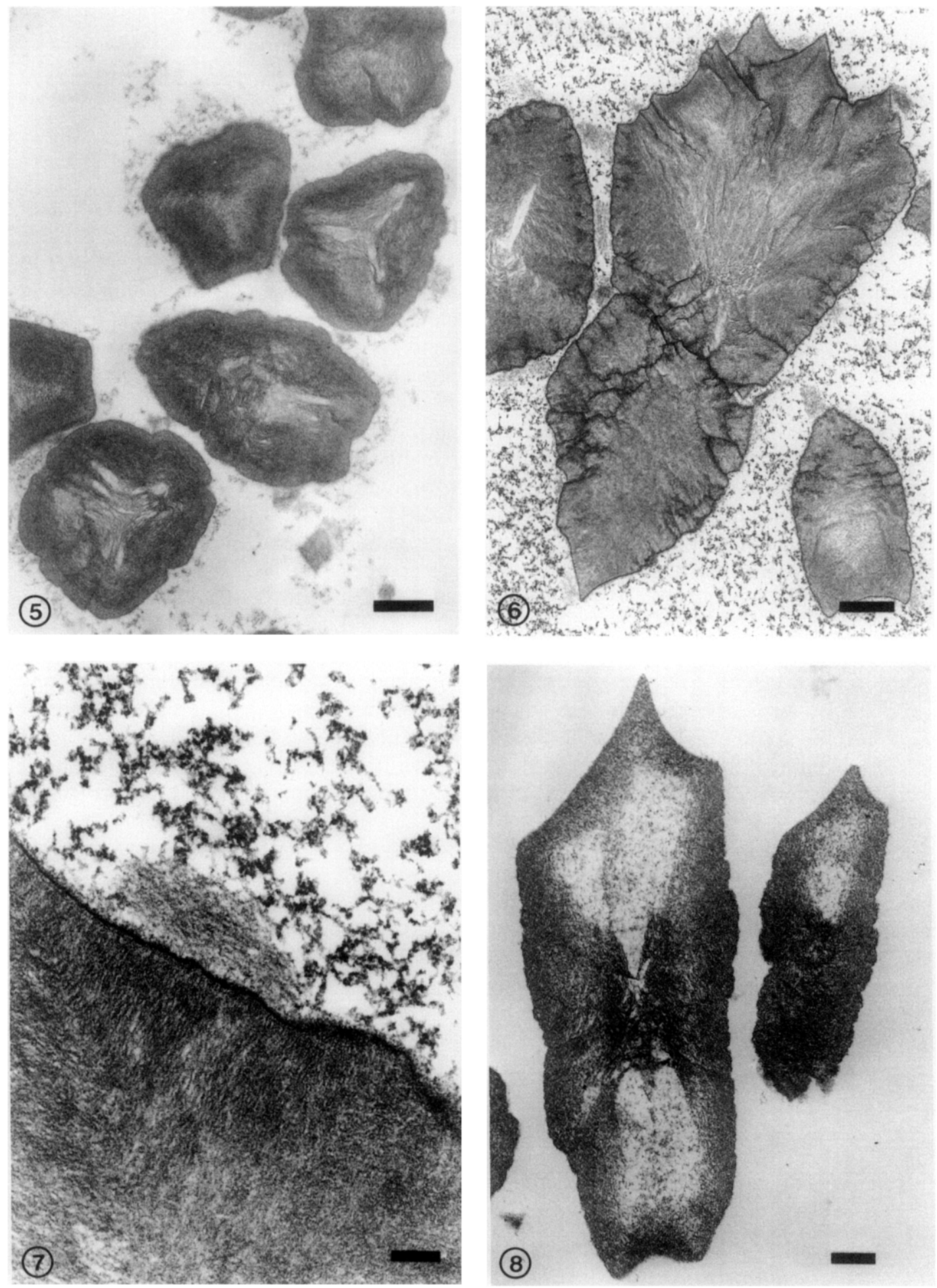
the primary fixative followed by overnight decalcification in formic acid (TA/FA) results in preservation of the otoconial core and peripheral zone, and the external lamina is remarkably well retained (Figs. 6 and 7). There is a fine line of organic material internal to and in register with the external lamina. This is most evident at the end faces (Fig. 7). The cores have distinct borders but are more electron lucent than after decalcification by EDTA or FA alone. The organic material of the peripheral zone is more uniformly distributed and more highly ordered than after other methods, and retains its segmented appearance. TA/FA demonstrates an additional organic material superficial to the otoconia. This material is granular as opposed to the fibrillar appearance of the otoconial membrane (Fig. 6). It is closely applied to the otoconia as well as the otoconial membrane, and appears to fill the membranous labyrinth.

\section{SDS Centrifugation}

Otoconia used for SDS-PAGE are microdissected prior to decalcification. Such samples are contaminated with otoconial membrane material (Fig. 1) and cellular debris unless centrifugation is employed. As shown in Fig. 8, samples sonicated and centrifuged in the presence of SDS prior to decalcification retain much internal organic material. It is evident, however, that material is lost from the peripheral zone and central core and that the external lamina often is not evident (compare with previous micrographs). By contrast, when fixation by glutaraldehyde is stopped by quenching with
$\mathrm{NaBH}_{4}$, subsequent decalcification with EDTA results in loss of the internal organic material (Figs. 9 and 10). The segmentally arranged material is retained and the surface lamina is well preserved.

\section{SDS Gel Electrophoresis}

The proteins separated from whole otoconial complexes are shown in Fig. 11, lane 9. At least 25 bands in a range between $\sim 16$ and $\sim 125 \mathrm{kDa}$ are evident. A major broad band of 90-100 kDa (band a) and a prominent narrow band of $\sim 56 \mathrm{kDa}$ (band $\mathrm{b}$ ) are present. Samples sonicated and centrifuged in the presence of SDS (see Fig. 9) show partial purification of some of these components (Fig. 11, lanes 7 and 8). Proteins with approximate molecular weights of 33 (band e), 40 (band d), 45 (band c), and a wide band of protein in the range of 90-100 are present in the SDS pellet (lane 8) while the $56-\mathrm{kDa}$ band is more prevalent in the supernatant (lane 7). The wide band overlaps several narrow bands which remain in the supernatant after SDS treatment (lane 7). Sonication and centrifugation in the absence of SDS fails to separate these proteins (lanes 10 and 11).

After cross-linking the proteins with glutaraldehyde, quenching with $\mathrm{NaBH}_{4}$, the pellet yields a pattern similar to that obtained from the pellet following SDS centrifugation (Fig. 11, lane 5). These contain comparable bands, while the supernatant of the cross-linked sample has only a high molecular weight $(\sim 200)$ band at the top of the gel. This band is not present in the separated protcins of wholc otoconial complexes (lane

FIG. 9. Dissected otoconia cross-linked with $4 \%$ glutaraldehyde, quenched with $1 \% \mathrm{NaBH}_{4}$, and decalcified with $5 \%$ EDTA. The internal otoconial matrix is absent. Bar $=5.0 \mu \mathrm{m}$.

FIG. 10. Dissected otoconia treated as in Fig. 9. Bar $=1.0 \mu \mathrm{m}$.

FIG. 11. SDS-PAGE, 6-16.6\% gradient gel. The molecular weights of the standards are shown at the left. Lanes 1-8 are from one gel, and lanes 9-11 are from a gel run under identical conditions. [(lane), sample]: (1), molecular weight standards; (2), EDTA + glutaraldehyde supernatant; (3), EDTA + glutaraldehyde pellet; (4), glutaraldehyde quenched with $\mathrm{NaBH}_{4}+$ EDTA supernatant; (5), glutaraldehyde quenched with $\mathrm{NaBH}_{4}+$ EDTA pellet; (6), blank; (7), SDS-treated supernatant; (8), SDS-treated pellet; (9), whole otoconial complex; (10), centrifuged without SDS pellet; (11), centrifuged without SDS supernatant. (a), 90-100 kDa; (b), $56 \mathrm{kDa}$; (c), $45 \mathrm{kDa}$; (d), $40 \mathrm{kDa}$; (e), $33 \mathrm{kDa}$ bands from the otoconial matrix. 
PROTEINS OF RAT OTOCONIAL COMPLEX

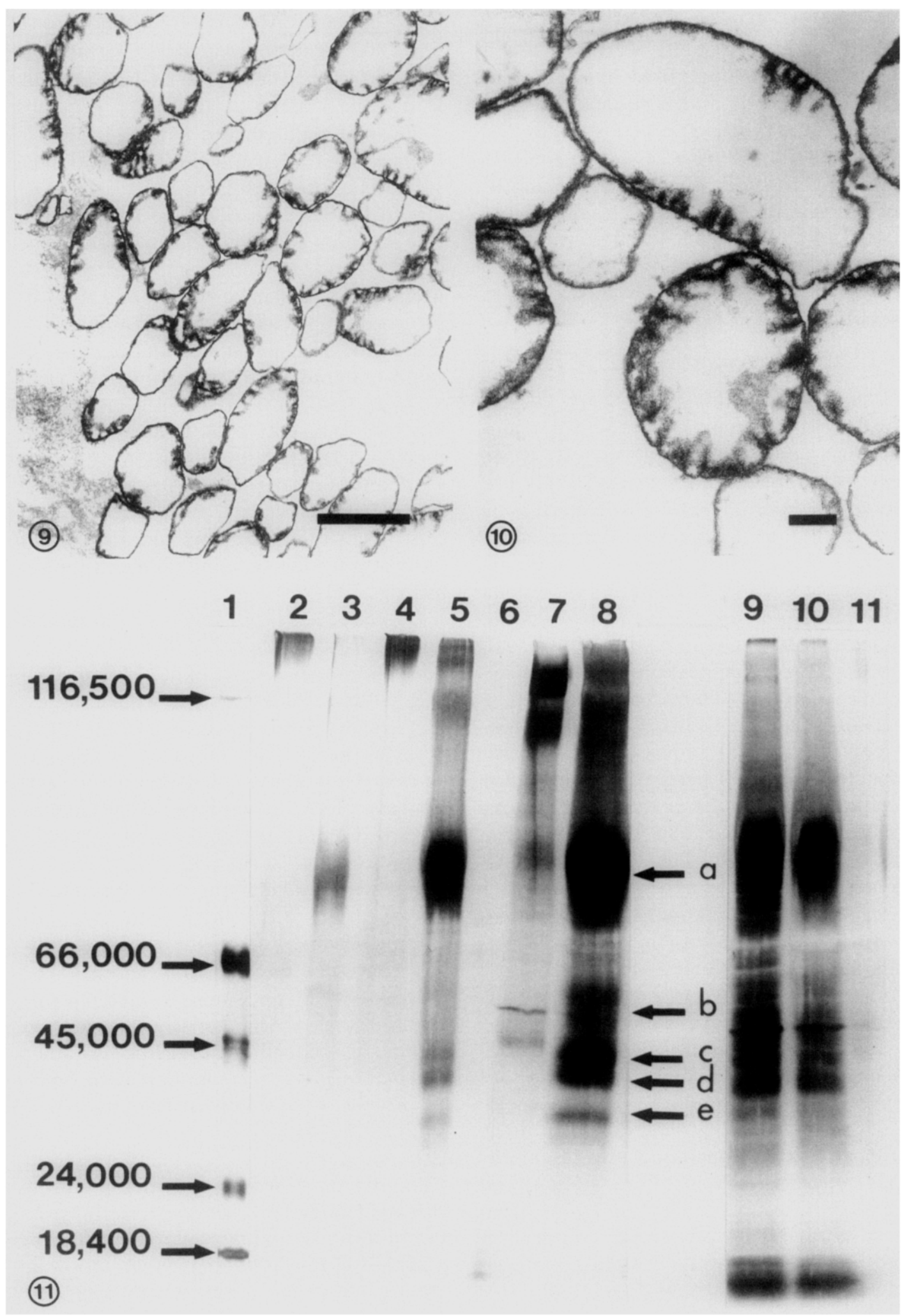


9). The $\sim 56-\mathrm{kDa}$ band, present in the SDScentrifuged supernatants, is reduced greatly in the glutaraldehyde cross-linked samples (compare lanes 4 and 7, supernatants, and lanes 5 and 8, pellets).

Decalcification with EDTA in the presence of glutaraldehyde reduces the number of bands in the pellet (Fig. 11, lane 3) compared with $\mathrm{NaBH}_{4}$-quenched samples (lane 5). A wide band at $\sim 90-100 \mathrm{kDa}$ is present, though it is reduced greatly in staining intensity.

\section{DISCUSSION}

The results of this study clearly indicate that the ultrastructural appearance of otoconia is dependent on the manner of decalcification. Glutaraldehyde/EDTA preserves the internal material yet removes the external lamina when decalcification is prolonged. Rapid decalcification of microdissected otoconia in this solution retains the external lamina and the segmented-appearing peripheral zonc. Howcver, in no case does EDTA decalcification preserve the organic material to the extent shown by inclusion of tannic acid in the primary fixative. This would indicate that organic materials from all parts of an otoconium (central core, peripheral zone, and external lamina) are EDTA soluble. Our electron microscopic evidence from the glutaraldehyde-quenching experiments, in which EDTA dissolved all material except that cross-linked, supports this idea. Degens (1976) showed that both EDTA-soluble and -insoluble fractions are present in the organic matrix of neogastropod shells.

Proteins from within the otoconia have been demonstrated using two different methods. Centrifugation in the presence of SDS or cross-linking the external proteins with glutaraldehyde results in the enrichment of a broad band of organic material of $M_{\mathrm{r}}=90-100 \mathrm{kDa}$. Electron microscopic results obtained from similarly prepared specimens demonstrate that this material must be within otoconia (Figs. 9 and 10).
There are also proteins of $33,40,45$, and $56 \mathrm{kDa}$ after these treatments. Variation is seen in the presence of the $56-\mathrm{kDa}$ protein, as discussed below.

The material in the broad band has a molecular weight slightly larger than that of the subunits of otolin isolated by Degens et al. (1969) from many species of fish otoliths. The subunits had a molecular weight range of $\sim 70-80 \mathrm{kDa}$. According to Degens et al., otolin has a molecular weight of $150 \mathrm{kDa}$, shown by molecular sieve techniques. Because otoconia have a small amount of organic material (four animals would yield $\sim 64 \mu \mathrm{g}$ total protein) sieving techniques have yet to be employed to assess whether the 90 - to $100-\mathrm{kDa}$ protein isolated here is a subunit of a larger aggregate. However, it does not appear to be a proteolytic product since phenylmethylsulfonyl fluoride (PMSF) did not alter the banding pattern (unpublished).

Degens et al. (1969) suggested that otolin serves as a template for otolith mineralization. The preponderance of the $\sim 90-$ to 100 $\mathrm{kDa}$ protein inside otoconia would suggest that it is involved in otoconial mineralization. Its precise role, however, is still unknown. Unpublished experiments in our laboratory have failed to identify a calciumbinding protein either by a mobility shift in the presence of calcium (Klee et al., 1979), or by differential staining with "Stainsall." This does not mean there is no calciumbinding protein. Rather, the staining methods may not be sensitive enough, or "Stainsall" may not stain the material differentially, as is true for $\left(\mathrm{Ca}^{2+}+\mathbf{M g}^{2+}\right)$ ATPase (Campbell et al., 1983). Another explanation is that there is no calcium-binding protein. The protein, instead, may bind or direct the seeding and growth of calcium carbonate crystallites into the mature form of the otoconia. We are continuing our efforts to identify a calcium-sequestering protein in these unique biominerals.

SDS-PAGE results show that otoconia contain at least three additional major, and 
several minor, internal proteins. These results differ somewhat from those of Ballarino (1985), who analyzed chick otoconia. Ballarino showed only one major protein of $97 \mathrm{kDa}$ to be present following SDS centrifugation.

Our results suggest that the $56-\mathrm{kDa}$ protein may be on the surface of otoconia and at interfaces of subunits. Glutaraldehyde cross-linked, $\mathrm{NaBH}_{4}$-quenched specimens examined ultrastructurally retained the external lamina and lost nearly all the internal organic material. Some material outlining segments remained. In comparably treated samples, the $56-\mathrm{kDa}$ band is reduced greatly in the pellet and is absent in the supernatant. The cross-linked material would have a high molecular weight. The band at the top of the gel in Fig. 11, lane 5, is this cross-linked material. Sonication and centrifugation with SDS removes much of the external lamina of otoconia and destroys their segmented appearance (Fig. 8). Likewise, the $56-\mathrm{kDa}$ protein is reduced in the otoconial pellet following this SDS treatment (Fig. 11, lane 8 ) but is prevalent in the supernatant. These results taken together suggest that the $56-\mathrm{kDa}$ protein may be closely associated with the otoconial surface.

The precise biochemical nature of the materials separated by SDS-PAGE is unknown. Results from EM samples treated with tannic acid would indicate the presence of anionic complex carbohydrates. Tannic acid forms conjugates through interactions with ionizable hydroxyl and carboxylic acid groups which are numerous in glycosaminoglycans (GAGs) or proteoglycans (PGs) (Simionescu and Simionescu, 1976). Vilstrup and Jensen (1954) determined that shark endolymph contained large amounts of the GAG, hyaluronic acid. A similar GAG may have been demonstrated with TA/FA as the granular material superficial to the otoconia (Fig. 6). Recent evidence for proteoglycan inhibition of mineralization in cartilage (Dziewiatkowski and Majznerski, 1985) suggests a role of the complex car- bohydrate demonstrated here. The surface protein obviously retains tannic acid and may function to inhibit the growth of otoconia.

Research efforts to identify the organic material within the otoconial membrane histochemically have yielded various results. Some authors have shown the presence of glycoprotein based on periodic acid Schiff (PAS) staining (Wislocki and Ladman, 1955; Belanger, 1960; Igarashi and Alford, 1969; Ross, 1973). Belanger (1953, 1956, 1960), using iron adsorption and metachromatic staining with toluidine blue, indicated the presence of glycosaminoglycan which may (Belanger, 1960) or may not (Vilstrup and Jensen, 1954) be sulfated. Shrader et al. (1973) and Trune and Lim (1983), from their work on the pallid mutant mouse, support the idea that proteoglycan is present. Belanger (1960) concluded that the otoconia have a protein fraction of neutral and acidic sulfated GAGs which are concentrated peripherally, while the otolithic membrane contains these in different proportions and is not mineralized.

In an attempt to determine whether glycoproteins or GAGs (proteoglycans) are present, Ross et al. (1985) analyzed rat otoconia separated by centrifugation for total sugar content using high-performance liquid chromatography (HPLC). Gil-Loyzaga et al. (1985) used fluorescent lectin binding to demonstrate the sugars present in otoconia. The glycans demonstrated by these methods are known to be present in both glycoproteins and GAGs. It is reasonable to assume that both proteoglycan(s) and glycoprotein(s) are present in otoconial complexes. Compositional analysis of the individual bands eluted from SDS-PAGE gels should begin to resolve the nature of the components of the various parts of the otoconia and the otoconial membrane. Perhaps then their functional significance will become more clear.

This work was supported by NASA Grant NAG 
2-325. We thank Kathleen M. Donovan for editorial corrections of this manuscript.

\section{REFERENCES}

Anderson, N. L., And Anderson, N. G. (1978) Anal. Biochem. 85, 341-354.

Ballarino, J. (1985) Thesis, Cornell University, Ithaca, NY.

BELANGER, L. F. (1953) Science 118, 520-521.

BELANGER, L. F. (1956) Science 123, 1074.

Belanger, L. F. (1960) in Sognnaes, R. F. (Ed.), Calcification in Biological Systems, Amer. Assoc. Adv. Sci., Washington, DC.

Campbell, K., Maclennen, D. H., and Jorgensen, A. O. (1983) J. Biol. Chem. 258, 11267-11273.

Carlstrom, D., Engstrom, H., and HJorth, S. (1953) Laryngoscope 63, 1052-1057.

Degens, E. T. (1976) Top. Curr. Chem. 64, 1-112.

Degens, E. T., Deyser, W. G., ANd Haedrich, R. L. (1969) Mar. Biol. 2, 105-113.

DzIEWIATKOWSKI, D. D., AND MAJZNERSKI, L. L. (1985) Calcif. Tissue Int. 37, 560-564.

Gil-Loyzaga P., RAymond, J., ANd Gabrion, J. (1985) Hear. Res. 18, 269-272.

HeNle, J. (1873) Quoted by Rudinger in Stricker's Manual of Human and Comparative Histology, Vol. 3, p. 122, New Sydenham Society, London.

Heukeshoven, J., AND Dernick, R. (1985) Electrophoresis 6, 103-112.

IGARASHI, M., AND ALFORD, B. R. (1969) Acta Otolaryngol. 68, 420-426.

IGARASHI, M., AND KANDA, T.(1969) Acta Otolaryngol. 68, 43-52.

IPPolito, E., LA Velle, S., AND Pedrini, V. (1981) Stain Technol. 56, 367-372.

Klee, C. B., Crouch, T. H., And Krinks, M. H. (1979) Proc. Natl. Acad. Sci. USA 76, 6270-6273.
LAEMMLI, U. K. (1970) Nature (London) 227, 680-685. LiM, D. J. (1973) Ann. Otol. 82, 23-35.

Lindeman, H. H. (1969) Adv. Anat. Embryol. Cell Biol. 42, 1-113.

Marco, J., Sanchez-Fernandez, J. Ma., and RIVera-Pomar, J. M. (1971) Acta Otolaryngol. 71, 1-8.

Nakahara, H., and Bevelander, G. (1979) Anat. Rec. 193, 233-242.

Ross, M. D. (1973) Anat. Rec. 175, 429.

Ross, M. D. (1979) Adv. Otol. Rhinol. Laryngol. 25, 26-33.

Ross, M. D. (1983) Adv. Space Res. 3, 179-190.

Ross, M. D., And Pote, K. G. (1984) Philos. Trans. R. Soc. London B 304, 445-452.

Ross, M. D., Pote, K. G., Cloke, P. L., AND Corson, C. (1980) Physiologist (Suppl), S129-S130.

Ross, M. D., Pote, K. G., AND Perini, F. (1985) in Drescher (Ed.), Auditory Biochemistry, pp. 500 514, Thomas, Springfield, IL.

Ross, M. D., Pote, K. G., Rarey, K. E., and Verma, L. M. (1981) Ann. N.Y. Acad. Sci. 374, 808-819.

Salamat, M. S., Ross, M. D., and Peacor, D. R. (1980) Ann. Olol. Rhinol. Laryngol. 89, 229-238.

Sanchez-Fernandez, J. Ma., Marco, J., RIvera-Pomar, J., and Delgado, R. M. (1972) Acta Otolaryngol. 73, 267-269.

Shrader, R. E., Erway, L. C., and Hurley, L. S. (1973) Teratology 8, 257-266.

Simionescu, N., ANd Simionescu, M. (1976) J. Cell Biol. 70, 622-633.

Spurr, A. R. (1969) J. Ultrastruct. Mol. Struct. Res. 26, 31-43.

Trune, D. R., AND Lim, D. J. (1983) Amer. J. Otolaryngol. 4, 261-272.

Vilstrup, T., and Jensen, C. E. (1954) Ann. Otol. Rhinol. Laryngol. 63, 151-163.

Wislocki, G. B., AND Ladman, A. J. (1955) J. Anat. 89, 3-12. 